\title{
IUFOST2006/1388 Processes Innovation for Bioactive Dairy Ingredients
}

\author{
J. Wan, M. Coventry and C. Versteeg \\ Food Science Australia, 671 Sneydes Road, Werribee, Victoria, 3030 Melbourne, Australia \\ jason.wan@csiro.au
}

Value-added dairy ingredients with demonstrated biological properties have attracted a great attention in recent years due to their potential applications in functional foods and nutraceuticals. These ingredients include lactoferrin, lactoperoxidase, colostrum powders and peptides from milk proteins. The biological properties include anti-bacterial, anti-viral, immunomodulation, angiotensin-I-converting-enzyme (ACE) inhibition, anticarcinogenic and growth factor-like activities. Most of these bioactive dairy ingredients are milk proteins or their derivatives, and their biological properties can be ablated during conventional heat processing. The emerging non-thermal processing technologies, including high pressure processing (HPP) and pulsed electric field (PEF), offer a potential alternative to the existing heat preservation processes without loss of biological properties of these ingredients. The presentation will focus on the use of these emerging processing technologies for bioactive functional food applications, including recent studies on microbial inactivation of dairy pathogens and spoilage organisms and preservation of heat-sensitive bioactive compounds in milk using the PEF technology. 